\title{
PRÉSENCE DE TAENIA HYDATIGENA PALLAS, 1766 CHEZ MACACUS CYNOMOLGUS L.
}

\author{
Par M. ISOBÉ
}

Le laboratoire de parasitologie a reçu, il y a quelque temps, un lot d'helminthes recueillis par M. Houdemer, vétérinaire-major à Hanoi. Parmi ceux-ci se trouvent des ténias provenant d'un singe, Macacus cynomolgus L., dont le $\mathrm{D}^{r}$ Ch. Joyeux a bien voulu me confier l'étude.

Ces cestodes appartiennent à deux espèces :

$1^{\circ}$ Bertiella conferta (Meyner, 1895), assez fréquent chez les macaques.

$2^{\circ}$ La seconde espèce peut être ainsi caractérisée. La longueur du ver, très contracté, atteint 55 centimètres, elle comprend 460 segments. La largeur maxima est de 4 millimètres. Par suite de la contraction, le cou est inexistant, et les premiers anneaux se voient immédiatement en arrière de la tête globuleuse. D'abord plus larges que longs, ils sont ensuite plus longs que larges en arrivant à maturité.

Le scolex est armé de deux couronnes, portant chacune 16 crochets, soit 32 en tout. Ils ont la forme caractéristiquc des crochets du genre Tænia. Voici leurs dimensions, exprimées en $\mu$.

Grandes crechets

Longueur totale ............. 198

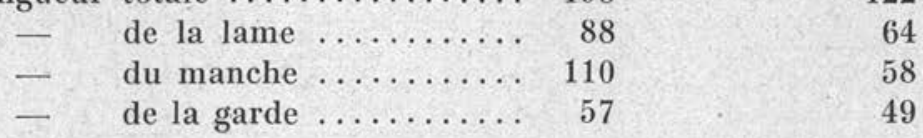

122

64

58

49

La longueur de la garde est prise de l'extrémité à la face opposée du crochet.

Les axes des ventouses ovalaires sont de 300 et $200 \mu$, soit à peu près $250 \mu$ de diamètre pour des ventouses arrondies.

Les organes génitaux présentent la structure caractéristique observée dans les genres Tænia et Multiceps. En raison de la contraction du ver, ils n'ont pu être examinés par transparence, il a été nécessaire de les étudier en coupes sériées.

Annales de Parasitologie, T. II, N ${ }^{\circ}$ 3. - Juillet 1924, p. 236. 
Les pores génitaux alternent irrégulièrement ; dans les anneaux jeunes, on distingue déjà l'ébauche des conduits génitaux décrivant une courbe vers la partie postérieure du proglottis.

Les anneaux sexués contiennent des testicules remplissant presque tout le proglottis à l'exception de la partie postéro-médiane qu'occupent les organes femelles. La poche du cirre mesure $380 \mu$ de long sur $140 \mu$ de largeur maxima. Le vagin décrit une sinuosité assez marquée au niveau du vaisseau ventral. On sait que ce caractère est indiqué par M. Hall (1) pour distinguer les genres Tænia et Multiceps. Malheureusement il n'est pas toujours facile à constater. L'ovaire est bilobé, le vitellogène étalé à la partie postérieure.

Les branches utérines se développent de bonne heure. Le tronc médian, longitudinal, émet bientôt des branches latérales nombreuses qui envahissent tout l'anneau. Elles sont bourrées d'œufs. Dans les derniers anneaux, elles sont dissociées et les øufs sont épars dans le parenchyme. Ces derniers sont du type incomplet. L'embryon est difficilement visible et je n'ai pu distinguer ses crochets. La coque interne mesure $27 \mu$ de diamètre, la coque moyenne $34 \mu$. L'intervalle compris entre les deux est fortement réfringent.

Reste à déterminer ce cestode. Il s'agit évidemment d'un Trnia ou d'un Multiceps. On connait des représentants de ces cestodes chez l'homme, chez quelques oiseaux et surtout chez les carnivores. Je parle ici du genre Tænia, sensu stricto, et non au sens des auteurs anciens, dont un certain nombre d'espèces continuent à figurer sous la rubrique Tænia en attendant leur révision et leur classement dans d'autres genres.

D'autre part, les cestodes connus chez les singes, notamment chez les macaques, sont surtout des anoplocéphalidés, spécialement des Bcrtiella. Nous avons donc à choisir entre deux hypothèses : ou bien il s'agit d'une nouvelle espèce du genre Tænia, lequel n'était pas encore connu chez ces singes ; ou bien c'est un Tænia connu, parasitant normalement d'autres animaux et égaré chez un primate. Nous adoptons cette seconde hypothèse, nous basant sur les raisons suivantes :

$1^{\circ}$ Macacus cynomolgus est un animal très utilisé dans les laboratoires. Un grand nombre d'autopsies en ont déjà été faites ; jamais on n'a signalé de Tænia, sensu stricto chez lui, mais toujours les cestodes habituels aux macaques.

$2^{\circ}$ La présence d'un Tænia chez un primate peut à la rigueur se comprendre pour un animal en captivité, ayant son régime alimen.

(1) M.-C. Har.t. The adult taenioid of dogs and cats of related carnivores in north america. Proc. U. S. nat. mus., 55, 1919. 
taire et, par conséquent, son chimisme intestinal complètemen! modifié.

Nous laissons de côté les anciennes cspèces douteuses attribuées au genre Tænia; nous négligeons aussi les espèces décrites chez les oiseaux, notamment Tænia diaphana Fuhr., 1908, des lariformes et $T$. heteracantha Fuhr., 1906, des accipitres, ayant des crochets de dimensions analogues à celles de notre espèce, mais parasitant des hôtes zoologiquement très éloignés des singes et d'une répartition géographique différente. Il nous semble plus logique de chercher parmi les cestodes communément rencontrés chez l'homme et les carnivores. La détermination, d'après les caractères des crochets, les œufs, l'aspect général des organes génitaux, nous conduit à Tænia hydatigena Pallas, 1776 (syn. Tænia marginata Batsch, 1786), parasite fréquent du chien.

La longueur relativement faible du ver est un caractère en faveur de son habitat accidentel.

La larve de ce cestode, Cysticercus tenuicollis Rud., 1810, parasite normal de divers ruminants (mouton, chèvre) a déjà été trouvée occasionnellement chez plusieurs singes; à ma connaissance, la forme adulte n'avait pas encore été signalée chez ces animaux.

\section{RÉSUMÉ}

Tænia hydatigena Pallas, parasite du chien, a été trouvé chez Macacus cynomolgus $\mathrm{L}$.

Laboraloire de Parasitologie de la Faculté de médecine de Paris. 